\title{
Tailor-made enhanced recovery programme for older patients
}

\author{
A. Deytrikh ${ }^{1} \cdot$ S. Tou ${ }^{1} \cdot$ R. Bergamaschi ${ }^{1}$
}

Received: 17 August 2015/Accepted: 17 September 2015/Published online: 26 September 2015

(C) Springer-Verlag Italia Srl 2015

Enhanced recovery after surgery (ERAS) has become the gold standard of care for surgical patients across a range of specialities. Developed by Kehlet and Wilmore many years after the first successful colorectal resection in 1833 by Reybard of Lyons, the majority of data for the use and application of ERAS currently relate to colorectal surgery. ERAS consists of a range of techniques such as preoperative carbohydrate loading, small incision surgery, reduced use of opioid analgesia, early post-operative mobilisation, and early enteral feeding to achieve improved recovery.

As seven of ten patients diagnosed with colorectal cancer in the UK are aged $>65$ [1], the focus around ERAS is now shifting towards its application in the older population. Currently, there is no standardisation between centres of the various parameters of the ERAS programme, and they have remained, on the whole, unaltered in the application towards older patients undergoing colorectal surgery.

Whether there is scope for adaption of the ERAS programme for use with older patients or continue with its generic application is a topic now worth addressing.

Older patients undergoing colorectal surgery are clearly at higher risk of post-operative complications such as pulmonary infection, urinary tract infection, post-operative heart failure, and delirium, compared with their younger counterparts. A recent systematic review found that ERAS reduces the occurrence of these complications in older patients [2]. Questions remained regarding the ability of

R. Bergamaschi

rcmbergamaschi@gmail.com

1 Division of Colorectal Surgery, T19-04 Health Sciences Center, State University of New York, Stony Brook, NY 11794-8191, USA older patients undergoing colorectal surgery to adhere to the ERAS protocol to the same degree as younger patients. However, a recent study found that patients $>60$ years of age closely adhered to the ERAS protocol better than or as well as those patients $<60$ years of age [3]. Despite this, it is not known which elements of the ERAS protocol have the greatest impact on patient outcomes, and less still, which of these elements contribute to superior outcomes for older patients, who differ, both physiologically and psychosocially, from younger patients undergoing colorectal resection. Great emphasis has been placed on length of stay when evaluating the effectiveness of ERAS. For younger patients, this seems reasonable as discharge home and return to baseline functioning is likely. However, there is limited evidence as to whether the same can be said for older patients. It is unclear what proportion of older patients are discharged directly back to their original environment, and which need to first undertake a further interim placement or rehabilitation before they return home. Moreover, one study found that older patients, who met the criteria for safe discharge, remained in hospital for a further 3-5 days after this date [4]. This may be due to limited availability of social support in the community, patient anxieties, and an unwillingness of surgeons to discharge their older patients in as timely a fashion as they may be inclined to with the younger population. More recently, functional status after surgery is gaining ground as a key outcome measure. Relying on length of stay as an outcome measure for older patients after major surgery is disingenuous. A recent study went beyond the assessment of length of stay when qualifying the effectiveness of the ERAS protocol for older patients; it compared pre- and post-operative functional status in individuals undergoing major colorectal resections, and found that for $83 \%$ of patients, post-operative function was at least as good as 
preoperative function [5]. Other centres have addressed the concept of prehabilitation with the aim of maintaining or improving functional status in older patients post-operatively. One study compared prehabilitation, based on resistance activities, nutritional counselling, and relaxation exercises, with rehabilitation in patients undergoing colorectal resection. Patients who had undergone prehabilitation had a significant improvement in functional exercise capacity, and returned to their functional baseline postoperatively [6]. More recently, a Delphi survey of colorectal surgeons found that exercise training should form part of preoperative care. However, there was no consensus on whether this type of exercise programme would be deliverable in many healthcare settings [7].

Further evaluation of older patient outcomes beyond discharge is needed. Consideration of both disease absence and preserved functional ability following major surgery, in order to maintain physical and social independence for older patients, now needs attention.

\section{Compliance with ethical standards}

Conflict of interest The authors declare that they have no conflict of interest.

Ethical approval This article does not contain any studies with human participants or animals performed by any of the authors.

Informed consent For this type of study, formal consent is not required.

\section{References}

1. Cancer Research UK. Bowl cancer incidence statistics. http:// www.cancerresearchuk.org/health-professional/cancer-statistics/ statistics-by-cancer-type/bowel-cancer/incidence\#heading-One. Accessed 15 June 2015

2. Bagnall NM, Malietzis G, Kennedy RH, Athanasiou T, Faiz O, Darzi A (2014) A systematic review of enhanced recovery care after colorectal surgery in elderly patients. Colorectal Dis 16:947-956

3. Bagnall NM, Malietzis G, Athanasiou T, Faiz O, Darzi A (2014) Elderly patients can adhere to and benefit from enhanced recovery care after colorectal surgery. Colorectal Dis 16(Suppl 2):20-21

4. Rumstadt B, Guenther N, Wendling P et al (2009) Multimodal perioperative rehabilitation for colonic surgery in the elderly. World J Surg 33:1757-1763

5. Wang Z, Tan KY, Tan P (2013) Functional outcomes in elderly adults who have undergone major colorectal surgery. J Am Geriatr Soc 61:2249-2250

6. Gillis C, Li C, Lee L et al (2014) Prehabilitation versus rehabilitation: a randomized control trial in patients undergoing colorectal resection for cancer. Anaesthesiology 121:937-947

7. Boereboom CL, Williams JP, Leighton P, Lund JN (2015) Forming a consensus opinion on exercise prehabilitation in elderly colorectal cancer patients: a Delphi study. Tech Coloproctol 19:347-354 